\title{
Variational Iteration Method for Solving System of Fractional Order Ordinary Differential Equations
}

\author{
Nabaa N. Hasan ${ }^{1}$, Fadhel S.Fadhel ${ }^{2}$ \\ ${ }^{1}$ (Department of Mathematics, College of Sciences, Al-Mustansiriyah University, Iraq) \\ ${ }^{2}$ (Department of Mathematics and Computer Applications, College of Science, Al-Nahrain University, Iraq)
}

\begin{abstract}
This paper presents approximate solutions for system of linear and nonlinear fractional order differential equations using the variational iteration method. The fractional derivatives are described in the Caputo sense, because it allows traditional initial and boundary conditions to be included in the formulation of the problem. The solutions of our models equations are calculated in the form of convergent series with easily computable components. Also, we prove the variational iteration formula and its convergence to the exact solution for solving system of fractional order differential equations. Some examples are solved as an illustration to the method, in order to show the accuracy of the method an comparison with results obtained by differential transform method and Adomian decomposition method given in author literatures .
\end{abstract}

Keywords: System of fractional differential equations, variational iteration method, Caputo fractional derivative.

\section{Introduction}

Deriving the iteration formula, proving its convergence to the exact solution and studying the approximate solution of Volterra and Fredholm integro-differential equation by using the variational iteration method (VIM) are given in [1]. Revised variational iteration method for solving system of ordinary differential equations used in [2]. Differential transform method used to approximate analytical solutions for system of fractional differential equations have been discussed in [3]. Homotopy analysis method used to derive the solution of fractional integro-differential equation is also introduced in [4]. Variational iteration method for solving KDV equation which arises in various physical phenomena related to physical and applied sciences is given in [5]. A reliable framework to solve the initial and boundary value problems of Bratu-type which are widely applicable in fuel ignition of the combustion theory and heat transfer is given in [6].

The main purpose of this paper is to derive the variational iteration formula and its convergence for solving system of fractional order ordinary differential equations (ODE). as well as some illustrative examples are given.

\section{Definitions And Preliminaries}

In this section, some fundamental and primitive concepts related to this paper are given for completeness purpose of this work.

Definition 2.1, [7]: A real function $\mathrm{f}(\mathrm{x}), \mathrm{x}>0$, is said to be in the space $\mathrm{C}_{\mu}, \mu \in \mathrm{R}$ if there exists a real number $p>\mu$, such that $f(x)=x^{p} f_{1}(x)$, where $f_{1}(x) \in C[0, \infty)$, and it is said to be in the space $C_{\mu}^{m}$ if and only if $\mathrm{f}^{(\mathrm{m})} \in \mathrm{C}_{\mu}, \mathrm{m} \in \mathrm{N}$.

Definition 2.2, [8]: The Riemann-Lioville fractional integral operator of order $\alpha>0$, of a function $f \in C_{\mu}, \mu \geq$ -1 , is defined as

$J^{\alpha} \mathrm{f}(\mathrm{x})=\frac{1}{\Gamma(\alpha)} \int_{0}^{\mathrm{x}}(\mathrm{x}-\mathrm{t})^{\alpha-1} \mathrm{f}(\mathrm{t}) \mathrm{dt}, \quad \alpha>0, \quad x>0$

Properties of the operator $\mathrm{J}^{\alpha}$ can be found in [1], we mention only the following: For $\mathrm{f} \in \mathrm{C}_{\mu}, \mu \geq-1, \alpha, \beta \geq$ 0 and $\gamma>-1$ :

$\mathrm{J}^{0} \mathrm{f}(\mathrm{x})=\mathrm{f}(\mathrm{x})$

$\mathrm{J}^{\alpha} \mathrm{J}^{\beta} \mathrm{f}(\mathrm{x})=\mathrm{J}^{\alpha+\beta} \mathrm{f}(\mathrm{x})$

$\mathrm{J}^{\alpha} \mathrm{x}^{\gamma}=\frac{\Gamma(\gamma+1)}{\Gamma(\alpha+\gamma+1)} \mathrm{x}^{\alpha+\gamma}$

Definition 2.3, [8]: The fractional derivative of $\mathrm{f}(\mathrm{x})$ in the Caputo sense is defined as

$D_{*}^{\alpha} f(x)=J^{m-\alpha} D^{m} f(x)=\frac{1}{\Gamma(m-\alpha)} \int_{0}^{x}(x-t)^{m-\alpha-1} f^{(m)}(t) d t$ 
for $\mathrm{m}-1<\alpha \leq \mathrm{m}, \mathrm{m} \in \mathrm{N}, \mathrm{x}>0, f \in \mathrm{C}_{-1}^{\mathrm{m}}$

Lemma (2.1), [7]: If $m-1<\alpha \leq \mathrm{m}, \mathrm{m} \in \mathrm{N}$ and $\mathrm{f} \in \mathrm{C}_{\mu}^{\mathrm{m}}, \mu \geq-1$, then

$D_{*}^{\alpha}{ }^{\alpha} f(x)=f(x)$

$J^{\alpha} D_{*}^{\alpha} f(x)=f(x)-\sum_{k=0}^{m-1} f^{(k)}\left(0^{+}\right) \frac{x^{k}}{k !}, x>0$

\section{The Variational Iteration Formula For Solving System Of Fractional Order Ode}

According to the variational iteration method, consider the following differential equation:

$\mathrm{L}_{\mathrm{u}}+\mathrm{N}_{\mathrm{u}}=\mathrm{f}(\mathrm{t})$

where $\mathrm{L}$ is a linear operator, $\mathrm{N}$ a non-linear operator and $\mathrm{f}(\mathrm{t})$ is the source inhomogeneous term. A correction functional is given as follow:

$\mathrm{u}_{\mathrm{n}+1}(\mathrm{t})=\mathrm{u}_{\mathrm{n}}(\mathrm{t})+\int_{0}^{\tau} \lambda(\tau)\left\{\mathrm{Lu}_{\mathrm{n}}(\tau)+\mathrm{N} \tilde{u}_{\mathrm{n}}(\tau)-\mathrm{f}(\tau)\right\} \mathrm{d} \tau$

where $\lambda$ is a general Lagrangian multiplier, which can be identified optimally via the variational theory, the second term on the right is called the correction and $\tilde{u}_{n}$ is considered as a restricted variation, i.e., $\delta \tilde{u}_{n}=0$.

Theorem (3.1): For the generalized fractional differential equations, one can have the variational iteration formula

$u_{n+1}(t)=u_{n}(t)+\int_{0}^{\tau} \lambda(t, \tau)\left\{D^{\alpha} u_{n}+u_{n}(\tau)+N \tilde{u}_{n}(\tau)-f(\tau)\right\} d \tau, \quad \alpha>0$

where the function $\lambda(\mathrm{t}, \tau)=\frac{(-1)^{\mathrm{k}}(\tau-\mathrm{t})^{\mathrm{k}-1}}{(\mathrm{k}-1) !}, \mathrm{k}=\Gamma^{\alpha}{ }_{\urcorner}$, is a Lagrange multiplier for any order $\alpha$, where $\left.\Gamma^{\alpha}\right\urcorner$ refers to the least positive integer greater than $\alpha$.

for, $0<\alpha_{i} \leq 1$, the Lagrange multiplier can be explicitly identified as $\lambda(t, \tau)=-1$

The proof of this theorem is given in [9].

In this paper, we consider the following system of fractional differential equations:

$D_{*}^{\alpha_{i}} x_{i}(t)=f_{i}\left(t, x_{1}, x_{2}, \ldots, x_{m}\right), \quad i=1,2, \ldots, m, m \in N, 0<\alpha_{i} \leq 1$

where $f_{i}$ are given continuous functions $D_{*}^{\alpha_{i}}$ is the Caputo derivative of $x_{i}$ of order $\alpha_{i}$, subject to the initial conditions:

$\mathrm{x}_{1}(0)=\mathrm{c}_{1}, \mathrm{x}_{2}(0)=\mathrm{c}_{2}, \ldots, \mathrm{x}_{\mathrm{m}}(0)=\mathrm{c}_{\mathrm{m}}$

The variational iteration formula for solving the above system may be derived as in the next theorem.

Theorem (3.2): Consider the system of fractional differential equations eq.(1). Then the related variational iteration formula is given by:

$x_{i, n+1}(t)=x_{i, n}(t)-I^{\alpha_{i}}\left\{D_{*}^{a_{i}} x_{i, n}(t)-f_{i}\left(t, x_{1, n}(t), x_{2, n}(t), \ldots, x_{m, n}(t)\right)\right\}$

where the subscript $\mathrm{n}$ denotes is the $\mathrm{n}^{\text {th }}$ approximation, $\mathrm{I}^{\mathrm{d}_{\mathrm{i}}}$ is the Riemann-Liouville's fractional integral of order $\alpha_{\mathrm{i}}$ and $\mathrm{D}_{*}^{\alpha_{\mathrm{i}}}$ is the Caputo derivative of $\mathrm{x}_{\mathrm{i}}$ of order $\alpha_{\mathrm{i}}, \quad 0<\alpha_{\mathrm{i}} \leq 1, \mathrm{i}=0,1, \ldots, \mathrm{m}$.

Proof: From system eq.(1) multiply by a general Lagrange multiplier $\lambda$ yields to:

$\lambda_{\mathrm{i}}\left\{\mathrm{D}_{*}^{\mathrm{a}_{\mathrm{i}}} \mathrm{x}_{\mathrm{i}}(\mathrm{t})-\mathrm{f}_{\mathrm{i}}\left(\mathrm{t}, \mathrm{x}_{1}(\mathrm{t}), \mathrm{x}_{2}(\mathrm{t}), \ldots, \mathrm{x}_{\mathrm{m}}(\mathrm{t})\right)\right\}=0, \mathrm{i}=1,2, \ldots, \mathrm{m}, \mathrm{m} \in \mathrm{N}, 0<\alpha_{\mathrm{i}} \leq 1$

Now take $I^{\alpha_{i}}$ to the both sides of system eq. (3)

$\mathrm{I}^{\alpha_{\mathrm{i}}} \lambda_{\mathrm{i}}\left\{\mathrm{D}_{*}^{\alpha_{\mathrm{i}}} \mathrm{x}_{\mathrm{i}}(\mathrm{t})-\mathrm{f}_{\mathrm{i}}\left(\mathrm{t}, \mathrm{x}_{1}(\mathrm{t}), \mathrm{x}_{2}(\mathrm{t}), \ldots, \mathrm{x}_{\mathrm{m}}(\mathrm{t})\right)\right\}=0, \mathrm{i}=1,2, \ldots, \mathrm{m}, \mathrm{m} \in \mathrm{N}, 0<\alpha_{\mathrm{i}} \leq 1$

and then the correction functional for system eq.(4) will be read as follows:

$\mathrm{x}_{\mathrm{i}, \mathrm{n}+1}(\mathrm{t})=\mathrm{x}_{\mathrm{i}, \mathrm{n}}(\mathrm{t})+\mathrm{I}^{\mathrm{\alpha}_{\mathrm{i}}} \lambda_{\mathrm{i}}\left\{\mathrm{D}_{*}^{\alpha_{\mathrm{i}}} \mathrm{x}_{\mathrm{i}, \mathrm{n}}(\mathrm{t})-\mathrm{f}_{\mathrm{i}}\left(\mathrm{t}, \mathrm{x}_{1, \mathrm{n}}(\mathrm{t}), \mathrm{x}_{2, \mathrm{n}}(\mathrm{t}), \ldots, \mathrm{x}_{\mathrm{m}, \mathrm{n}}(\mathrm{t})\right)\right\}$

Hence by theorem (3.1), substituting $\lambda_{\mathrm{i}}=-1, \mathrm{i}=1,2, \ldots, \mathrm{m}$ into the correction functional system eq.(5), will results the iteration formula eq.(2).

Theorem (3.3): Let $x_{i} \in\left(C^{2}[0, T],\|\cdot\|_{\infty}\right)$ be the exact solution of the fractional differential system eq.(1) and $x_{i, n} \in C^{2}[0, T]$ be the obtained solution of the sequence defined by eq.(2). If $E_{i, n}(t)=x_{i, n}(t)-x_{i}(t)$ and $f_{i}$ in eq.(1) satisfies Lipschitz condition with constants $\mathrm{L}_{\mathrm{i}}$, such that $\mathrm{L}_{\mathrm{i}}<\Gamma\left(\alpha_{\mathrm{i}}\right)$, then the sequence of approximate solutions $x_{i, n}, n=0,1, \ldots$; converges to the exact solution $x_{i}$.

Proof: Consider the system of fractional differential equations eq.(1):

$D_{*}^{\alpha_{i}} x_{i}(t)=f_{i}\left(t, x_{1}, x_{2}, \ldots, x_{m}\right), \quad i=1,2, \ldots, m, m \in N, 0<\alpha_{i} \leq 1$

$\mathrm{x}_{1}(0)=\mathrm{c}_{1}, \mathrm{x}_{2}(0)=\mathrm{c}_{2}, \ldots, \mathrm{x}_{\mathrm{m}}(0)=\mathrm{c}_{\mathrm{m}}$

where the approximate solution using the VIM is given by:

$x_{i, n+1}(t)=x_{i, n}(t)-I^{\alpha_{i}}\left\{D_{*}^{\alpha_{i}} x_{i, n}(t)-f_{i}\left(t, x_{1, n}(t), x_{2, n}(t), \ldots, x_{m, n}(t)\right)\right\}$

since $x_{i}$ is the exact solution of fractional differential system, hence: 
$x_{i}(t)=x_{i}(t)-I^{\alpha_{i}}\left\{D_{*}^{\alpha_{i}} x_{i}(t)-f_{i}\left(t, x_{1}(t), x_{2}(t), \ldots, x_{m}(t)\right)\right\}$

hence, subtracting $x_{i}$ from $x_{i, n+1}$ yields to:

$E_{i, n+1}=E_{i, n}-I^{\alpha_{i}}\left\{D_{*}^{\alpha_{i}} E_{i, n}-f_{i}\left(t, x_{1, n}, x_{2, n}, \ldots, x_{m, n}\right)-f_{i}\left(t, x_{1}, x_{2}, \ldots, x_{m}\right)\right\}$

$$
=E_{i, n}(t)-E_{i, n}(t)-E_{i, n}(0)+I^{\alpha_{i}}\left\{f_{i}\left(t, x_{1, n}, x_{2, n}, \ldots, x_{m, n}\right)-f_{i}\left(t, x_{1}, x_{2}, \ldots, x_{m}\right)\right\}
$$

where $E_{i, n}(0)=x_{i, n}(0)-x_{i}(0)$, with $x_{i, n}(0)=x_{i}(0)$ then $E_{i, n}(0)=0$ therefore:

$E_{i, n+1}=I^{\alpha_{i}}\left\{f_{i}\left(t, x_{1, n}, x_{2, n}, \ldots, x_{m, n}\right)-f_{i}\left(t, x_{1}, x_{2}, \ldots, x_{m}\right)\right\}$

$$
=\frac{1}{\Gamma\left(\alpha_{i}\right)} \int_{0}^{t}(t-s)^{\alpha_{i}-1}\left\{f_{i}\left(t, x_{1, n}, x_{2, n}, \ldots, x_{m, n}\right)-f_{i}\left(t, x_{1}, x_{2}, \ldots, x_{m}\right)\right\} d s
$$

now, take the maximum norm of the two sides of $E_{i, n+1}$, will give:

$$
\begin{aligned}
\left\|E_{i, n+1}(t)\right\|_{\infty} & =\left\|\frac{1}{\Gamma\left(\alpha_{i}\right)} \int_{0}^{t}(t-s)^{\alpha_{i}-1}\left\{f_{i}\left(t, x_{1, n}, x_{2, n}, \ldots, x_{m, n}\right)-f_{i}\left(t, x_{1}, x_{2}, \ldots, x_{m}\right)\right\}\right\|_{\infty} \\
\leq & \frac{1}{\Gamma\left(\alpha_{i}\right)} \int_{0}^{t}\|t-s\|_{\infty}^{\alpha_{i}-1}\left\|f_{i}\left(t, x_{1, n}, x_{2, n}, \ldots, x_{m, n}\right)-f_{i}\left(t, x_{1}, x_{2}, \ldots, x_{m}\right)\right\|_{\infty} d s \\
\leq & \frac{1}{\Gamma\left(\alpha_{i}\right)} \int_{0}^{t} \max _{s \in[0, t]}|t-s|^{\alpha_{i}-1} L_{i}\left\|x_{i, n}(s)-x_{i}(s)\right\|_{\infty} d s \\
= & \frac{1}{\Gamma\left(\alpha_{i}\right)} \int_{0}^{t} t^{\alpha_{i}-1} L_{i}\left\|E_{i, n}(s)\right\|_{\infty} d s
\end{aligned}
$$

Hence:

$$
\left\|E_{i, n+1}(t)\right\|_{\infty} \leq \frac{L_{i}}{\Gamma\left(\alpha_{i}\right)} t^{\alpha_{i}-1} \int_{0}^{t}\left\|E_{i, n}(s)\right\|_{\infty} d s, \quad \forall n=0,1, \ldots
$$

Now if $n=0$, then:

$$
\begin{aligned}
\left\|\mathrm{E}_{\mathrm{i}, 1}(\mathrm{t})\right\|_{\infty} & \leq \frac{\mathrm{L}_{\mathrm{i}}}{\Gamma\left(\alpha_{\mathrm{i}}\right)} \mathrm{t}^{\alpha_{\mathrm{i}}-1} \int_{0}^{\mathrm{t}}\left\|\mathrm{E}_{\mathrm{i}, 0}(\mathrm{~s})\right\|_{\infty} \mathrm{ds} \\
& \leq \frac{\mathrm{L}_{\mathrm{i}}}{\Gamma\left(\alpha_{\mathrm{i}}\right)} \mathrm{t}^{\alpha_{\mathrm{i}}-1} \int_{0}^{\mathrm{t}} \max _{\mathrm{s} \in[0, \mathrm{t}]}\left|\mathrm{E}_{\mathrm{i}, 0}(\mathrm{~s})\right|_{\infty} \mathrm{ds} \\
& =\frac{\mathrm{L}_{\mathrm{i}}}{\Gamma\left(\alpha_{\mathrm{i}}\right)} \mathrm{t}^{\alpha_{\mathrm{i}}} \max _{\mathrm{s} \in[0, \mathrm{t}]}\left|\mathrm{E}_{\mathrm{i}, 0}(\mathrm{~s})\right|
\end{aligned}
$$

Also for $\mathrm{n}=1$, we have:

$$
\begin{aligned}
& \left\|E_{i, 2}(t)\right\|_{\infty} \leq \frac{L_{i}}{\Gamma\left(\alpha_{i}\right)} t^{\alpha_{i}-1} \int_{0}^{t}\left\|E_{i, 1}(s)\right\|_{\infty} d s \\
& \quad \leq \frac{L_{i}}{\Gamma\left(\alpha_{i}\right)} t^{\alpha_{i}-1} \int_{0}^{t} \frac{L_{i}}{\Gamma\left(\alpha_{i}\right)} s^{\alpha_{i}} \max _{s \in[0, t]}\left|E_{i, 0}(s)\right| d s \\
& \leq\left(\frac{L_{i}}{\Gamma\left(\alpha_{i}\right)}\right)^{2} \frac{t^{2 \alpha_{i}}}{\alpha_{i}+1} \max _{s \in[0, t]}\left|E_{i, 0}(s)\right|
\end{aligned}
$$

Similarly, for $\mathrm{n}=2$, then:

$$
\begin{aligned}
\left\|E_{i, 3}(t)\right\|_{\infty} & \leq \frac{L_{i}}{\Gamma\left(\alpha_{i}\right)} t^{\alpha_{i}-1} \int_{0}^{t}\left\|E_{i, 2}(s)\right\|_{\infty} d s \\
& \leq \frac{L_{i}}{\Gamma\left(\alpha_{i}\right)} t^{\alpha_{i}-1} \int_{0}^{t}\left(\frac{L_{i}}{\Gamma\left(\alpha_{i}\right)}\right)^{2} \frac{s^{2 \alpha_{i}}}{\alpha_{i}+1} \max _{s \in[0, t]}\left|E_{i, 0}(s)\right| d s \\
& =\left(\frac{L_{i}}{\Gamma\left(\alpha_{i}\right)}\right)^{3} t^{\alpha_{i}-1} \max _{s \in[0, t]}\left|E_{i, 0}(s)\right| \int_{0}^{t} \frac{s^{2 \alpha_{i}}}{\alpha_{i}+1} d s \\
& =\left(\frac{L_{i}}{\Gamma\left(\alpha_{i}\right)}\right)^{3} t^{\alpha_{i}-1} \max _{s \in[0, t]}\left|E_{i, 0}(s)\right| \frac{t^{2 \alpha_{i}+1}}{\left(\alpha_{i}+1\right)\left(2 \alpha_{i}+1\right)}
\end{aligned}
$$




$$
=\left(\frac{L_{i}}{\Gamma\left(\alpha_{i}\right)}\right)^{3} \frac{t^{3 \alpha_{i}}}{\left(\alpha_{i}+1\right)\left(2 \alpha_{i}+1\right)} \max _{s \in[0, t]}\left|E_{i, 0}(s)\right|
$$

$\vdots$

$$
\begin{aligned}
& \left\|\mathrm{E}_{\mathrm{i}, \mathrm{n}}(\mathrm{t})\right\| \leq\left(\frac{\mathrm{L}_{\mathrm{i}}}{\Gamma\left(\alpha_{\mathrm{i}}\right)}\right)^{\mathrm{n}} \frac{\mathrm{t}^{\mathrm{n} \alpha_{\mathrm{i}}}}{\left(\alpha_{\mathrm{i}}+1\right)\left(2 \alpha_{\mathrm{i}}+1\right) \ldots\left((\mathrm{n}-1) \alpha_{\mathrm{i}}+1\right)} \max _{s \in[0, \mathrm{t}]}\left|\mathrm{E}_{\mathrm{i}, 0}(\mathrm{~s})\right| \\
& \leq\left(\frac{\mathrm{L}_{\mathrm{i}}}{\Gamma\left(\alpha_{\mathrm{i}}\right)}\right)^{\mathrm{n} \alpha_{\mathrm{i}}} \frac{}{\left(\alpha_{\mathrm{i}}+1\right)\left(2 \alpha_{\mathrm{i}}+1\right) \ldots\left((\mathrm{n}-1) \alpha_{\mathrm{i}}+1\right)} \max _{s \in[0, \mathrm{t}]}\left|\mathrm{E}_{\mathrm{i}, 0}(\mathrm{~s})\right|
\end{aligned}
$$

and since $\mathrm{L}_{\mathrm{i}}<\Gamma\left(\alpha_{\mathrm{i}}\right)$, so as $\mathrm{n} \rightarrow \infty$, we have $\left\|\mathrm{E}_{\mathrm{i}, \mathrm{n}}(\mathrm{t})\right\|_{\infty} \rightarrow 0$, i.e., $\mathrm{x}_{\mathrm{i}, \mathrm{n}} \rightarrow \mathrm{x}_{\mathrm{i}}$

\section{Illustrative Examples}

To illustrate the application of theorem (3.2) and theorem (3.3) for solving system of fractional differential equations, three examples are given. All the results are calculated by using the microcomputer software MathCad 15

First, we start with linear system of fractional order ODE's.

Example 4.1: Cconsider the following system of two linear fractional differential equations

$\mathrm{D}_{*}^{\beta} \mathrm{x}(\mathrm{t})=\mathrm{x}(\mathrm{t})+\mathrm{y}(\mathrm{t})$

$D_{*}^{\gamma} \mathrm{y}(\mathrm{t})=-\mathrm{x}(\mathrm{t})+\mathrm{y}(\mathrm{t})$

$\mathrm{x}(0)=0, \mathrm{y}(0)=1$

for comparison, the exact solution for this example with $\beta=\gamma=1$ is given by [3]

$\mathrm{x}(\mathrm{t})=\mathrm{e}^{\mathrm{t}} \sin (\mathrm{t})$

$y(t)=e^{t} \cos (t)$

Using the vriational iteration formula given by eq.(2) with values of $\beta=1$ and $\gamma=1$ we have system of ordinary differential equations that may be solved using six iterations to get the following results:

$x(t)=t+t^{2}+\frac{t^{3}}{3}-\frac{t^{5}}{30}-\frac{t^{6}}{90}-\frac{t^{7}}{630}$

$y(t)=1+t-\frac{t^{3}}{3}-\frac{t^{4}}{6}-\frac{t^{5}}{30}+\frac{t^{7}}{630}$

The obtained results for this example are the same results obtained by S. Vedat, M. Shaher in, [3]

Now for $\beta=0.7$ and $\gamma=0.9$, and proceeding similarly, we get the following variational iteration formulas for $\mathrm{n}=0,1, \ldots$

$\mathrm{x}_{\mathrm{n}+1}=\mathrm{x}_{\mathrm{n}}-\mathrm{I}^{0.7}\left\{\mathrm{D}^{0.7} \mathrm{x}_{\mathrm{n}}-\mathrm{x}_{\mathrm{n}}-\mathrm{y}_{\mathrm{n}}\right\}$

$\mathrm{y}_{\mathrm{n}+1}=\mathrm{y}_{\mathrm{n}}-\mathrm{I}^{0.9}\left\{\mathrm{D}^{0.9} \mathrm{y}_{\mathrm{n}}+\mathrm{x}_{\mathrm{n}}-\mathrm{y}_{\mathrm{n}}\right\}$

by initial conditions eq.(7) and use the properties of lemma (2.1), for $n=0$ we have:

$\mathrm{x}_{1}=0-\mathrm{I}^{0.7}\left\{\mathrm{D}^{0.7}(0)-0-1\right\}=\frac{\mathrm{t}^{0.7}}{\Gamma(1.7)}$

$\mathrm{y}_{1}=1-\mathrm{I}^{0.9}\left\{\mathrm{D}^{0.9}(1)+0-1\right\}=1+\frac{\mathrm{t}^{0.9}}{\Gamma(1.9)}$

also for $\mathrm{n}=1$ we have:

$$
\begin{aligned}
\mathrm{x}_{2} & =\frac{\mathrm{t}^{0.7}}{\Gamma(1.7)}-\mathrm{I}^{0.7}\left\{\mathrm{D}^{0.7}\left(\frac{\mathrm{t}^{0.7}}{\Gamma(1.7)}\right)-\frac{\mathrm{t}^{0.7}}{\Gamma(1.7)}-1-\frac{\mathrm{t}^{0.9}}{\Gamma(1.9)}\right\} \\
& =\frac{\mathrm{t}^{0.7}}{\Gamma(1.7)}+\frac{\mathrm{t}^{1.4}}{\Gamma(2.4)}+\frac{\mathrm{t}^{1.6}}{\Gamma(2.6)} \\
\mathrm{y}_{2} & =1+\frac{\mathrm{t}^{0.9}}{\Gamma(1.9)}-\mathrm{I}^{0.9}\left\{\mathrm{D}^{0.9}\left(1++\frac{\mathrm{t}^{0.9}}{\Gamma(1.9)}\right)+\frac{\mathrm{t}^{0.7}}{\Gamma(1.7)}-1-\frac{\mathrm{t}^{0.9}}{\Gamma(1.9)}\right\} \\
& =1-\frac{\mathrm{t}^{1.6}}{\Gamma(2.6)}+\frac{\mathrm{t}^{0.9}}{\Gamma(1.9)}+\frac{\mathrm{t}^{1.8}}{\Gamma(2.8)}
\end{aligned}
$$

Similarly, for $\mathrm{n}=2$ we have :

$$
\begin{aligned}
& \mathrm{x}_{3}(\mathrm{t})=\frac{\mathrm{t}^{0.7}}{\Gamma(1.7)}+\frac{\mathrm{t}^{1.4}}{\Gamma(2.4)}+\frac{\mathrm{t}^{1.6}}{\Gamma(2.6)}+\frac{\mathrm{t}^{2.1}}{\Gamma(3.1)}+\frac{\mathrm{t}^{2.5}}{\Gamma(3.5)} \\
& \mathrm{y}_{3}(\mathrm{t})=1+\frac{\mathrm{t}^{0.9}}{\Gamma(1.9)}-\frac{\mathrm{t}^{1.6}}{\Gamma(2.6)}+\frac{\mathrm{t}^{1.8}}{\Gamma(2.8)}-\frac{\mathrm{t}^{2.3}}{\Gamma(3.3)}-\frac{2 \mathrm{t}^{2.5}}{\Gamma(3.5)}
\end{aligned}
$$

For comparisons purpose, table (1) show the absolute error of the results of the proposed method with results is given by, S. Vedat, M. Shaher [3]. 
Table (1): The absolute error of the proposed method $x_{i}(t), y_{i}(t)$ and differential transform method $x(t), y(t)$

\begin{tabular}{|l|l|l|l|l|l|l|}
\hline $\mathrm{t}$ & $\left|\mathrm{x}(\mathrm{t})-\mathrm{x}_{1}(\mathrm{t})\right|$ & $\left|\mathrm{x}(\mathrm{t})-\mathrm{x}_{2}(\mathrm{t})\right|$ & $\left|\mathrm{x}(\mathrm{t})-\mathrm{x}_{3}(\mathrm{t})\right|$ & $\left|\mathrm{y}(\mathrm{t})-\mathrm{y}_{1}(\mathrm{t})\right|$ & $\left|\mathrm{y}(\mathrm{t})-\mathrm{y}_{2}(\mathrm{t})\right|$ & $\left|\mathrm{y}(\mathrm{t})-\mathrm{y}_{3}(\mathrm{t})\right|$ \\
\hline 0 & 0.000 & 0.000 & 0.000 & 0.000 & 0.000 & 0.000 \\
\hline 0.1 & 0.054 & $4.566 \times 10^{-3}$ & 0.000 & 0.012 & $3.771 \times 10^{-3}$ & 0.000 \\
\hline 0.2 & 0.159 & 0.021 & 0.000 & 0.040 & 0.02 & 0.000 \\
\hline 0.3 & 0.302 & 0.051 & 0.000 & 0.087 & 0.053 & 0.000 \\
\hline 0.4 & 0.482 & 0.097 & 0.000 & 0.153 & 0.106 & 0.000 \\
\hline 0.5 & 0.695 & 0.159 & 0.000 & 0.242 & 0.182 & 0.000 \\
\hline 0.7 & 1.222 & 0.24 & 0.000 & 0.354 & 0.283 & $1.332 \times 10^{-15}$ \\
\hline 0.8 & 1.536 & 0.339 & 0.000 & 0.492 & 0.411 & $1.332 \times 10^{-15}$ \\
\hline 0.9 & 1.882 & 0.457 & 0.000 & 0.658 & 0.568 & $2.442 \times 10^{-15}$ \\
\hline 1 & 2.260 & 0.596 & $1.332 \times 10^{-15}$ & 0.852 & 0.755 & $2.665 \times 10^{-15}$ \\
\hline
\end{tabular}

Example 4.2: Let us consider the following system of two nonlinear fractional differential equations

$D_{*}^{\beta} x(t)=2 y^{2}(t)$

$\mathrm{D}_{*}^{\gamma} \mathrm{y}(\mathrm{t})=\mathrm{tx}(\mathrm{t})$

$\mathrm{x}(0)=0, \mathrm{y}(0)=1$

for values $\beta=1$ and $\gamma=1, \mathrm{x}(\mathrm{t})$ and $\mathrm{y}(\mathrm{t})$ are obtained after 3 iterations as follows:

$\mathrm{x}_{3}(\mathrm{t})=2 \mathrm{t}+2 \frac{\mathrm{t}^{4}}{3}+8 \frac{\mathrm{t}^{7}}{63}$

$y_{3}(t)=1+2 \frac{t^{3}}{3}+\frac{t^{6}}{9}+4 \frac{t^{9}}{189}$

The obtained results for this example are the same results obtained by S. Vedat, M. Shaher in, [3]

$x(t)=2 t+4 \frac{t^{10}}{105}+2 \frac{t^{4}}{3}+2 \frac{t^{7}}{21}$

$y(t)=1+2 \frac{t^{3}}{3}+\frac{t^{6}}{9}+4 \frac{t^{9}}{189}$

for values $\beta=0.5$ and $\gamma=0.4$, for $n=0$ the results as follows:

$\mathrm{x}_{1}(\mathrm{t})=2 \frac{\mathrm{t}^{0.5}}{\Gamma(1.5)}$

$\mathrm{y}_{1}(\mathrm{t})=1$

also for $\mathrm{n}=1$

$\mathrm{x}_{2}(\mathrm{t})=2 \frac{\mathrm{t}^{0.5}}{\Gamma(1.5)}$

$\mathrm{y}_{2}(\mathrm{t})=1+2 \frac{\Gamma(2.5)}{\Gamma(1.5) \Gamma(2.9)} \mathrm{t}^{1.9}$

Similarly for $\mathrm{n}=2$

$\mathrm{x}_{3}(\mathrm{t})=2 \frac{\mathrm{t}^{0.5}}{\Gamma(1.5)}+8 \frac{\Gamma(2.5)}{\Gamma(1.5) \Gamma(3.4)} \mathrm{t}^{2.4}$

$\mathrm{y}_{3}(\mathrm{t})=1+2 \frac{\Gamma(2.5)}{\Gamma(1.5) \Gamma(2.9)} \mathrm{t}^{1.9}$

For comparisons purpose, table (2) show the absolute error of the results of the proposed method with results is given by, S. Vedat, M. Shaher [3].

Table (2): The absolute error of the proposed method $x_{i}(t), y_{i}(t)$ and differential transform method $x(t), y(t)$

\begin{tabular}{|l|l|l|l|}
\hline $\mathrm{t}$ & $\left|\mathrm{x}(\mathrm{t})-\mathrm{x}_{2}(\mathrm{t})\right|$ & $\left|\mathrm{x}(\mathrm{t})-\mathrm{x}_{3}(\mathrm{t})\right|$ & $\left|\mathrm{y}(\mathrm{t})-\mathrm{y}_{2}(\mathrm{t})\right|$ \\
\hline 0 & 0 & 0 & 0 \\
\hline 0.1 & 0.016 & 0 & 0 \\
\hline 0.2 & 0.085 & 0 & 0 \\
\hline 0.3 & 0.224 & $1.554 \times 10^{-15}$ & 0 \\
\hline 0.4 & 0.446 & $2.22 \times 10^{-15}$ & 0 \\
\hline 0.5 & 0.763 & $3.109 \times 10^{-15}$ & $1.11 \times 10^{-15}$ \\
\hline 0.6 & 1.181 & $4.441 \times 10^{-15}$ & $1.776 \times 10^{-15}$ \\
\hline 0.7 & 1.710 & $6.217 \times 10^{-15}$ & $1.998 \times 10^{-15}$ \\
\hline 0.8 & 2.356 & $7.105 \times 10^{-15}$ & $3.109 \times 10^{-15}$ \\
\hline 0.9 & 3.126 & $9.77 \times 10^{-15}$ & $3.553 \times 10^{-15}$ \\
\hline 1 & 4.025 & $1.155 \times 10^{-15}$ & $4.441 \times 10^{-15}$ \\
\hline
\end{tabular}

Example 4.3: Consider the system of nonlinear fractional differential equations

$D_{*}^{\alpha} x(t)=x^{2}(t)+y(t)$ 
$D_{*}^{\beta} y(t)=y(t) \cos (x(x))$

$\mathrm{x}(0)=0, \mathrm{y}(0)=1$

where, $\alpha, \beta \in(0,1)$. To solve cos! $(\mathrm{x}(\mathrm{t}))$ we will use the series:

$\cos (\mathrm{x})=1-\frac{\mathrm{x}^{2}}{2 !}+\frac{\mathrm{x}^{4}}{4 !}-\mathrm{O}\left(\mathrm{x}^{6}\right)$

In view of the results obtained by Jafari and Daftardar-Gejji [10], this system has the solution:

$x(t)=\frac{t^{\alpha}}{\Gamma(\alpha+1)}+\frac{t^{\alpha+\beta}}{\Gamma(\alpha+\beta+1)}+\frac{\Gamma(2 \alpha+1) t^{3 \alpha}}{(\Gamma(\alpha+1))^{2} \Gamma(3 \alpha+1)}$

$y(t)=1+\frac{t^{\beta}}{\Gamma(\beta+1)}+\frac{t^{2 \beta}}{\Gamma(2 \beta+1)}-\frac{\Gamma(2 \alpha+1) t^{2 \alpha+\beta}}{(\Gamma(\alpha+1))^{2} \Gamma(2 \alpha+\beta+1)}$

For $\mathrm{n}=0$ the results as follows:

$\mathrm{x}_{1}(\mathrm{t})=\frac{\mathrm{t}^{\alpha}}{\Gamma(\alpha+1)}$

$\mathrm{y}_{1}(\mathrm{t})=1+\frac{\mathrm{t}^{\beta}}{\Gamma(\beta+1)}$

Similarly for $\mathrm{n}=1$

$\mathrm{x}_{2}(\mathrm{t})=\frac{\mathrm{t}^{\alpha}}{\Gamma(\alpha+1)}+\frac{\mathrm{t}^{\alpha+\beta}}{\Gamma(\alpha+\beta+1)}+\frac{\Gamma(2 \alpha+1) \mathrm{t}^{3 \alpha}}{(\Gamma(\alpha+1))^{2} \Gamma(3 \alpha+1)}$

$\mathrm{y}_{2}=1+\frac{\mathrm{t}^{\beta}}{\Gamma(\beta+1)}+\frac{\mathrm{t}^{2 \beta}}{\Gamma(2 \beta+1)}-\frac{\Gamma(2 \alpha+1) \mathrm{t}^{2 \alpha+\beta}}{2(\Gamma(\alpha+1))^{2} \Gamma(2 \alpha+\beta+1)}-\frac{\Gamma(2 \alpha+\beta+1) \mathrm{t}^{2 \alpha+2 \beta}}{2(\Gamma(\alpha+1))^{2} \Gamma(\beta+1) \Gamma(2 \alpha+2 \beta+1)}$

For comparisons purpose, table (3) show the absolute error of the results of the proposed method with results is given by, H. Jafari, V. Daftardar-Gejji [10].

Table (3): The absolute error of the proposed method $\mathrm{x}_{\mathrm{i}}(\mathrm{t}), \mathrm{y}_{\mathrm{i}}(\mathrm{t})$ and Adomian decomposition method $x(t), y(t)$

\begin{tabular}{|l|l|l|l|l|}
\hline $\mathrm{t}$ & $\left|\mathrm{x}(\mathrm{t})-\mathrm{x}_{1}(\mathrm{t})\right|$ & $\left|\mathrm{x}(\mathrm{t})-\mathrm{x}_{2}(\mathrm{t})\right|$ & $\left|\mathrm{y}(\mathrm{t})-\mathrm{y}_{1}(\mathrm{t})\right|$ & $\left|\mathrm{y}(\mathrm{t})-\mathrm{y}_{2}(\mathrm{t})\right|$ \\
\hline 0 & 0.000 & 0.000 & 0.000 & 0.000 \\
\hline 0.1 & 0.000 & 0.000 & 0.070 & 0.010 \\
\hline 0.2 & 0.000 & 0.000 & 0.114 & 0.024 \\
\hline 0.3 & 0.000 & 0.000 & 0.143 & 0.036 \\
\hline 0.4 & 0.000 & 0.000 & 0.158 & 0.045 \\
\hline 0.5 & 0.000 & 0.000 & 0.161 & 0.050 \\
\hline 0.6 & 0.000 & 0.000 & 0.155 & 0.051 \\
\hline 0.7 & 0.000 & 0.000 & 0.139 & 0.047 \\
\hline 0.8 & 0.000 & 0.000 & 0.115 & 0.037 \\
\hline 0.9 & 0.000 & 0.000 & 0.082 & 0.022 \\
\hline 1 & 0.000 & 0.000 & 0.042 & $1.434 \times 10^{-3}$ \\
\hline
\end{tabular}

\section{Conclusion}

In this paper, variational iteration method used for solving a system of fractional differential equations. The method provides the solution as infinite series of functions with easily computable components. The examples show that the results of the present method are in agreement with those obtained by differential transform method and Adomian decomposition method. The response functions of different force functions are obtained for different values of $\alpha(0<\alpha \leq 1)$, where $\alpha=1$ the system reduce to system of ordinary differential equations which give the exact solution.

\section{Refrences}

[1]. H. Mariam, Wadea, Variational Iteration Method for Solving Fractional Order Integro-Differential Equations, Msc thesis AlMustansirya University College of Education, 2012.

[2]. S. Elham, J. Hossein, A. Merich, Revised Variational Iteration Method for Solving System of Ordinary Differential Equations, an international Journal of Applications and Applied Mathematics, Special Issue No.1, 2010, 110-121.

[3]. S. Vedat, M. Shaher, Solving Systems of Fractional Differential Equations Using Differential Transform method, Journal of Computational and Applied Mathematics, 215, 2008, 142-151.

[4]. A. Fadi, E. Rawashdeh, H. Jaradat, Analytic Solution of Fractional Integro-Differential Equations, Journal of Mathematics and Computer Science Series, 38(1), 2011, 1-10.

[5]. S. Tauseef, M. Aslam, Variational Iteration Method for Solving Discrete KDV Equation, Blletin of the Institute of Mathematics Academia Sinica (New Series), 5(1), 2010, 69-73.

[6]. S. Tauseef, M. Aslam, Variational Iteration Method for Solving Initial and Boundary value problems of Bratu-type, Journal of Applications and Applied Mathematics, 3(1), 2008, 89-99.

[7]. M. Shaher, W. Rabha, Analytical Solutions of Fractional Oscillator by the Decomposition Method, International Journal of Pure and Applied Mathematics, 37(1), 2007, 119-131. 
[8]. E. A. Rawashdeh, Legendre Wavelets Method for Fractional Integro-Differential Equations, Journal of Applied Mathematical Sciences, 5(50), 2011, 2467-2474.

[9]. C. Guo, B. Dumitru, New Application of the Variational Iteration Method form Differential Equations to q-Fractional Difference Equations, Spring Open Journal, 2013, 1-16.

[10]. H. Jafari, V. Daftardar-Gejji , Solving a System of Nonlinear Fractional Differential Equations Using Adomian Decomposition, Journal of Computational and Applied Mathematics, 196, 2006, 644-651. 